\title{
Restricción del crecimiento intrauterino: una aproximación al diagnóstico, seguimiento y manejo
}

\author{
Laura Marcela Pimiento Infante ${ }^{1 a}$, Mónica Andrea Beltrán Avendaño. ${ }^{1}$ \\ ${ }^{1}$ Escuela de Medicina, Facultad de Salud. Departamento de Obstetricia y Ginecología, Universidad Industrial de San- \\ tander. Colombia.
} a Programa de Obstetricia y Ginecología, Escuela de Medicina, Facultad de Salud, Departamento de Obstetricia y Gine-
cología, Universidad Industrial de Santander. Colombia.

\section{RESUMEN}

Antecedentes: La restricción del crecimiento intrauterino (RCIU) es una entidad de origen multifactorial que puede ser causada por una gran variedad de patologías a nivel materno, fetal o placentario, y que representa altas tasas de morbimortalidad materna y perinatal. Es importante realizar un diagnóstico certero de esta patología con el fin de llevar a cabo un enfoque de seguimiento y de manejo que pueda disminuir todas las complicaciones asociadas a la enfermedad. Objetivo: Realizar una revisión detallada y actualizada de la etiopatogenia, criterios diagnósticos, seguimiento con ecografía Doppler y manejo de la restricción del crecimiento intrauterino. Método: Se realizó una búsqueda de literatura publicada en inglés y español en bases de datos como PubMed/MEDLINE, Ovid, MDconsult utilizando las palabras clave: restricción del crecimiento intrauterino, feto pequeño para la edad gestacional, circulación fetal, ecografía Doppler, además de la búsqueda de estadísticas relacionadas con RCIU, de los resultados obtenidos se seleccionaron 61 artículos. La información obtenida fue clasificada y utilizada como soporte para la realización de esta revisión. Resultados: Se presentan los estudios disponibles, las revisiones y artículos recomendados para la evaluación de pacientes con RCIU, adicionalmente aquellos que brindan nueva información con respecto al diagnóstico, evaluación de circulación fetal, seguimiento y manejo de esta entidad clínica. Conclusión: La restricción del crecimiento intrauterino es una patología que presenta altas tasas de morbimortalidad perinatal. La identificación temprana de estos fetos y el seguimiento adecuado mediante la evalución Doppler permiten disminuir los resultados adversos y las secuelas a corto y largo plazo.

\section{PALABRAS CLAVE: Restricción del crecimiento intrauterino, feto pequeño para la edad gestacional, circulación fetal, ecografía Doppler fetal}

\section{SUMMARY}

Background: Intrauterine growth restriction has a multifactorial origin and can be caused by a variety of pathologies in the mother, fetus or placenta, representing high rates of maternal and perinatal morbidity and mortality. Therefore, it is important to accurately diagnose this condition in order to focus in the follow and management, which can reduce the complications associated with the disease. Objective: To perform a detailed and up to date review of the etiology, diagnostic criteria, follow up with Doppler ultrasonography and management of intrauterine growth restriction. Methods: A literature search was done in English and Spanish in databases such us PubMed/MEDLINE, Ovid, MDconsult using the following key words: intrauterine growth restriction, small for gestational age fetus, fetal circulation, Doppler ultrasonography. Also national statistics for intrauterine growth restriction were used, obtaining 61 documents. The information obtained 
was classified and used in the development of this review. Results: The available studies are presented, and recommended revisions to the evaluation of patients with IUGR, additionally those that provide new information regarding the diagnosis, evaluation of fetal circulation, monitoring and management of this clinical entity. Conclusion: Intrauterine growth restriction is a pathology with a frequent diagnosis an represents high rates of perinatal morbidity and mortality. Early identification of these fetuses and adequate monitoring by Doppler evaluation allow reducing adverse outcomes and consequences in the short and long term.

\section{KEY WORDS: Intrauterine growth restriction, small for gestational age fetus, fetal circulation, fetal Doppler ultrasonography}

\section{INTRODUCCIÓN}

La restricción del crecimiento intrauterino (RCIU) es una patología de causas multifactoriales y manejo complejo para el obstetra. Para un feto el no alcanzar su potencial de crecimiento representa un aumento significativo del riesgo de morbimortalidad perinatal (1). Como no es posible determinar el potencial de crecimiento fetal, para su definición se utilizan tablas de crecimiento intrauterino, aceptándose como crecimiento normal el peso para la edad gestacional entre los percentiles 10 y 90 , como analizaremos posteriormente. Para evaluar el crecimiento fetal se utilizan elementos clínicos como la medición de la altura uterina y la estimación del peso fetal, complementado con la fetometría ultrasonográfica y la medición de flujos sanguíneos de ciertos territorios vasculares fetales. El objetivo principal de la detección prenatal de fetos con alteraciones del crecimiento es reducir la morbilidad y la mortalidad asociada con este problema empleando para tal fin en determinados momentos intervenciones ya establecidas.

La RCIU está asociada de forma indirecta con complicaciones perinatales a corto plazo como son la asfixia intraparto (explicada por la hipoxia crónica a la que son sometidos estos fetos sumado a la disminución transitoria del flujo sanguíneo placentario durante el parto) (2), hipoglucemia, hipotermia, policitemia, convulsiones, coagulopatías, sepsis, hiperbilirrubinemia y prematurez $(2,3)$, con un aumento significativo en las admisiones a las unidades de cuidados intensivos (RR: 3,4; IC95\%: 1,6$7,4)(4)$. Además de su relación con la prematurez, aquellos recién nacidos antes de las 32 semanas de gestación y que tienen RCIU, presentan mayor incidencia de complicaciones relacionadas con la prematurez, requieren soporte ventilatorio por periodos de tiempo más largos y fallecen en mayor cantidad que los prematuros con peso adecuado para la edad gestacional (4).

A largo plazo tiene impacto adverso en el desarrollo infantil presentándose alteraciones en el tono muscular, coeficientes intelectuales menores que aquellos infantes con peso adecuado, trastornos del comportamiento y emocionales $(5,6)$, y en la vida adulta se relaciona con disminución de la productividad económica y bajo peso al nacer de los hijos (4).

El objetivo de esta comunicación es realizar una revisión detallada y actualizada de la etiopatogenia, criterios diagnósticos, seguimiento con ecografía Doppler y manejo de la restricción del crecimiento intrauterino.

\section{METODOLOGÍA}

Se realizó una búsqueda de literatura publicada en inglés y español en bases de datos como PubMed/MEDLINE, Ovid, MDconsult utilizando las palabras clave: restricción del crecimiento intrauterino, feto pequeño para la edad gestacional, circulación fetal, ecografía Doppler, limitada a ensayo clínico, revisión, metaanálisis, revisiones sistemáticas. Se obtuvieron 125 resultados para los últimos 5 años, además de la búsqueda manual de publicaciones relacionadas, y se revisaron en total 61 incluyendo artículos con enfoque general sobre epidemiología, diagnóstico, fisiopatología, cambios hemodinámicos asociados, seguimiento y manejo, incluyendo información reportada a nivel Latinoamericano y de Colombia.

\section{DEFINICIONES}

La deficiencia en el crecimiento fetal implica una falla en el feto para alcanzar todo su potencial de crecimiento establecido genéticamente (7). Este potencial de crecimiento varía fisiológicamente de acuerdo a las características maternas como peso, talla, origen étnico, paridad, condición nutricional, además se ve afectado por otro tipo de factores como consumo de cigarrillo, trastornos hipertensivos, diabetes, y otras patología maternas, como también la prematuridad (8).

Se consideran fetos con RCIU los que presentan los siguientes parámetros $(1,9,10,11)$ :

- Crecimiento del feto por debajo del percentil 10 para la edad gestacional, acompañado de signos de compromiso fetal (anormalidades de la circulación feto-placentaria identificadas por Doppler) $(1,9,10)$. 
- Peso menor al percentil 3 para la edad gestacional $(1,9,10)$.

Los fetos pequeños para la edad gestacional son aquellos cuyo peso se encuentra entre los percentiles 3 y 10, con una valoración anatómica dentro de límites normales, pruebas de bienestar fetal satisfactorias y persistencia del crecimiento dentro de los mismos percentiles durante la gestación $(10,11)$.

\section{CLASIFICACIÓN DE LA RESTRICCIÓN DEL CRECIMIENTO INTRAUTERINO}

Actualmente se encuentra vigente la clasificación de la restricción según el grado de severidad y de acuerdo al momento de inicio $(7,8)$ :

- RCIU severo: Fetos que se encuentran con peso ultrasonográfico estimado por debajo del percentil 3 para la edad gestacional. Esta clasificación tiene peor pronóstico y aumento en la morbilidad y mortalidad perinatal.

- RCIU temprano: De aparición antes de la semana 32 de gestación, se ha encontrado relación estrecha con la prematuridad, preeclampsia y alteraciones a nivel placentario.

- RCIU tardío: Es la forma más frecuente, aparece después de la semana 32 , se encuentra también asociado a enfermedad placentaria, pero en menor grado que el grupo de RCIU temprano

\section{ETIOLOGÍA}

En general podemos dividir las causas de RCIU en factores maternos, fetales y placentarios.

\section{Factores maternos}

Trastornos hipertensivos: Se presentan hasta en un $30-40 \%$ de los embarazos complicados con RCIU $(12,13,14)$. La preeclampsia y la hipertensión crónica complicada con preeclampsia se han asociado con un aumento hasta de 4 veces el riesgo de obtener fetos pequeños para la edad gestacional (15-18).

Trastornos autoinmunes: Principalmente aquellos en los que hay compromiso vascular como el síndrome de anticuerpos antifosfolípidos (24\%) y el lupus eritematoso sistémico (12).

Trombofilias: La más estudiada ha sido el polimorfismo relacionado con el Factor $\mathrm{V}$ de Leiden. Un metaanálisis publicado en el 2005 por Howley y cols (19), revisó 10 estudios de casos y controles y encontró asociación entre la presencia de factor $\mathrm{V}$ de Leiden y restricción del crecimiento fetal (OR 2,7; IC95\%: 1,3-5,5).

Estilo de vida: El consumo de sustancias psicoactivas, el consumo de cigarrillo, alcohol y cocaína, se han asociado a RCIU (12,20-23).

Trastornos del ánimo: Se ha estudiado la asocia- ción entre depresión materna y RCIU concluyendo a favor del aumento del riesgo de RCIU, variando el efecto en función del grado de depresión, el estado socioeconómico, del diagnóstico y tratamiento de la depresión antes del embarazo (24).

Fármacos: Están incluidos los medicamentos antineoplásicos, anticonvulsivantes (fenitoína), beta bloqueadores (especialmente atenolol) y esteroides (corticoides) $(12,15)$.

Desnutrición: Dependiendo de la severidad de la deprivación de nutrientes en la madre y del trimestre en que se presente serán los resultados sobre el crecimiento fetal $(12,13)$.

\section{Factores fetales}

Aneuploidías: Aproximadamente el $7 \%$ de los casos de RCIU se han asociado con aneuploidías. El $90 \%$ de los fetos con trisomía 18 cursan con restricción del crecimiento, comparado con el $30 \%$ de aquellos con trisomía 21(12). La aparición temprana de restricción del crecimiento, se ha relacionado con trisomía 18 y $13(14,25)$.

Malformaciones: Más del $22 \%$ de los recién nacidos con malformaciones congénitas cursan con $\mathrm{RCIU}$. El riesgo aumenta desde el $20 \%$ cuando se presentan 2 defectos, hasta el $60 \%$ en quienes presentan 9 o más defectos. Las malformaciones que más se encuentran relacionadas con RCIU son: cardiacas (tetralogía de Fallot, corazón izquierdo hipoplásico, estenosis pulmonar y defectos del septo ventricular), anencefalia y defectos de la pared abdominal $(25,26)$.

Infección perinatal: Contribuyen con aproximadamente 5 a $10 \%$ de los casos cuando se trata de infecciones intrauterinas de origen viral (rubéola, citomegalovirus, VIH, varicela zoster) y de protozoarios (malaria, toxoplasmosis). La infección e inflamación subclínica (coriamnionitis diagnosticada por histología) puede llevar al resultado de restricción del crecimiento en el feto, así como la infección extragenital y la periodontal, si bien este último foco infeccioso es motivo de controversia $(27,28,29)$.

Prematuridad: Gardosi (30) y Bukowski y cols (31) han encontrado la asociación entre parto prematuro y RCIU. Así estos últimos publicaron un estudio de casos y controles en el que observaban que aproximadamente el $30 \%$ de los fetos que nacían antes de las 35 semanas de gestación tenían peso por debajo del percentil 10 comparado con el $4,5 \%$ de aquellos fetos nacidos a las 37 o más semanas de gestación (30).

Gestaciones múltiples: Aproximadamente hasta la semana 32 de gestación las curvas de crecimiento fetal se mantienen similares en embarazo simples y múltiples, posteriormente los fetos de embarazos múltiples muestran tendencia a la restricción del crecimiento y dependiente de la corionicidad $(20 \%$ en bicoriales, $30 \%$ en monocoriales) $(12,15)$. 


\section{Factores placentarios}

Las principales entidades asociadas a RCIU son placenta previa, infartos placentarios, vasculitis, arteria umbilical única, placenta circunvalada, inserción velamentosa del cordón, tumores placentarios, angiogénesis aberrante $(1,31)$.

\section{CAMBIOS HEMODINÁMICOS ASOCIADOS A RCIU}

Los cambios circulatorios que se observan en la $\mathrm{RCIU}$ de etiología placentaria se originan en el crecimiento y desarrollo inadecuado de la misma. En estos casos, la primera alteración que se identifica en el Doppler es el aumento de la pulsatilidad en las arterias uterinas. Si esta situación permanece, el feto se adapta con una modesta disminución en la tasa de crecimiento y gastará más energía en la circulación placentaria, sin compromiso a largo plazo $(32,33)$. A medida que se hace crónica la injuria, los mecanismos de adaptación fetal incluyen disminución del crecimiento somático, del tamaño del hígado y de los depósitos de grasa. Cuando estos cambios adaptativos son suficientes no hay cambios hemodinámicos sistémicos y pueden observarse solamente aumento en la resistencia placentaria $(32,33)$.

En los casos de RCIU, la fase inicial es de compensación, en la que ocurren cambios cardiovasculares no detectables en el Doppler; a medida que empeora el proceso, ocurre una cascada de cambios que pueden ser detectados clínicamente (32) y que discutiremos a continuación.

Arterias uterinas: A medida que la placenta invade la decidua del miometrio, las arterias sufren grandes modificaciones haciéndolas semejantes a vasos venosos. Estos cambios se encuentran bien establecidos al finalizar el primer trimestre, momento en el cual puede iniciar el seguimiento con Doppler de este vaso que en un embarazo normal muestran una onda con flujo alto durante la diástole. A medida que avanza el embarazo la disminución de la resistencia vascular se refleja en el incremento del flujo en diástole (Figura 1). Una onda anormal se caracteriza por altos índices de pulsatilidad y puede encontrarse alterada en presencia de una arteria umbilical normal (Figura 2) (13,32-35).

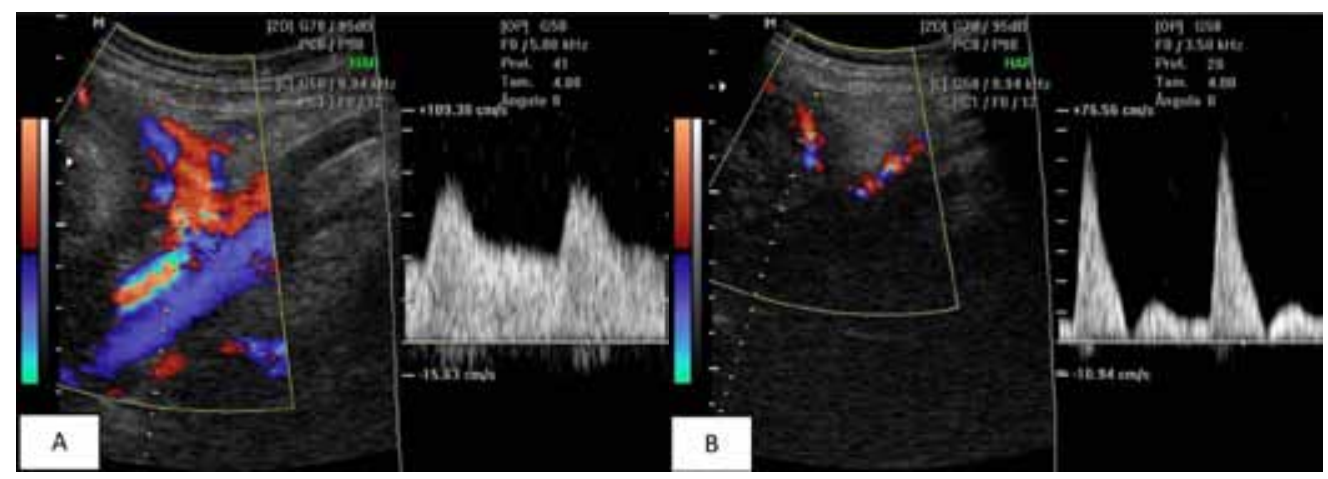

Figura 1. Doppler de arterias uterinas. A: Patrón de flujo normal en arteria uterina en el que se observa un patrón de flujo diastólico alto, no hay incisura. B: Patrón de flujo anormal en arteria uterina, en la que se observa incisura y flujo diastólico bajo.

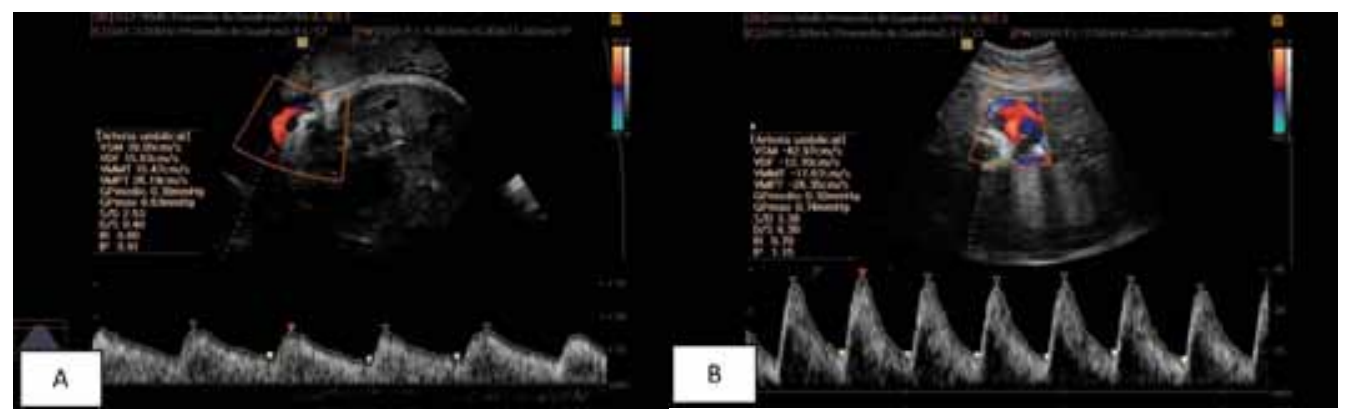

Figura 2. Doppler de arteria umbilical. A: Se observa el patrón de flujo normal en la arteria umbilical, hay flujo diastólico elevado, el índice de pulsatilidad y la relación S/D son bajos. B: Patrón de flujo anormal en la arteria umbilical con aumento de la resistencia. 
La alteración en la perfusión de la placenta en RCIU de inicio temprano comparado con fetos que tienen peso adecuado, es más pronunciada en el tercer trimestre de la gestación, por lo que la persistencia de alteraciones en las arterias uterinas en el final del embarazo tiene un impacto negativo en el desenlace del mismo $(36,37)$. Cuando es evaluada en fetos que crecen por debajo del percentil $10 \mathrm{y}$ que se encuentran a término, puede ser de utilidad como predictor de resultados adversos durante el trabajo de parto (estado fetal no satisfactorio, acidosis neonatal) (38).

La sensibilidad de la evaluación de las arterias uterinas para la identificación de resultados adversos es de $37,7 \%$ con especificidad de $70 \%$, valor predictivo positivo de 91,8 y valor predictivo negativo de $11,1 \%$ (39).

Arteria umbilical: Ha sido uno de los primeros vasos arteriales en ser estudiados en los casos de RCIU y es la única medida que proporciona información diagnóstica y pronóstico. La evidencia indica que su evaluación y seguimiento mejora los resultados perinatales con una reducción de $29 \%$ de las muertes perinatales (38).

En un embarazo normal se observa baja resistencia en el flujo feto-placentario y en gestaciones complicadas con RCIU se evidencian velocidades anormales en el flujo diastólico que se encuentran en relación con pobres pronósticos perinatales, principalmente el flujo reverso que puede presentarse cuando el lecho capilar placentario se encuentra reducido en más del $50 \%$ (13). La sensibilidad de la medición de los índices de arteria umbilical para identificación de resultados fetales adversos es de $64,4 \%$ con una especificidad de $80 \%$, valor predictivo positivo de $96,6 \%$ y valor predictivo negativo de $20 \%$ (39).

Los cambios en el tiempo se caracterizan por aumento de la pulsatilidad (Figura 2), ausencia del flujo de fin de diástole y finalmente en casos más severos flujo inverso en el final de diástole, estos dos últimos se presentan aproximadamente 1 semana antes de un deterioro agudo $(31,34,38)$.

Arteria cerebral media: Bajo condiciones normales la circulación cerebral es de alta impedancia (Figura 3). La respuesta a la hipoxia es la redistribución de flujo a órganos que tengan más demanda de oxígeno como el cerebro, miocardio y glándulas suprarrenales (40), esto resulta en oligoamnios por disminución del flujo sanguíneo renal y cambios a nivel de la circulación cerebral que se caracterizan por el aumento de la velocidad de fin de diástole. Esto se cuantifica en el Doppler como disminución en el índice de pulsatilidad de la arteria cerebral media al mismo tiempo que ocurren los cambios descritos previamente en la arteria umbilical, lo que se conoce como el fenómeno de redistribución de flujo (Figura 3) $(13,32,35)$.
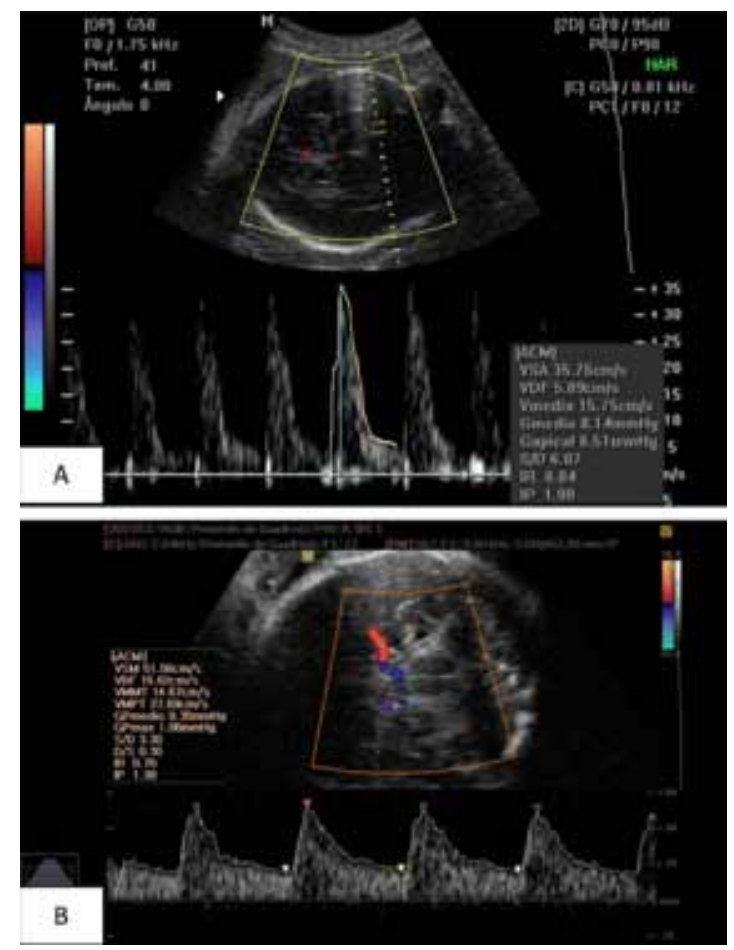

Figura 3. Doppler de arteria cerebral media. A: Flujo normal en arteria cerebral media con alta resistencia y bajos volúmenes diastólicos. B: Patrón de flujo anormal en arteria cerebral media con patrón de baja resistencia y volúmenes diastólicos altos.

La medición del flujo de la arteria cerebral media es valiosa para la identificación y la predicción de resultados perinatales adversos en los casos de RCIU de inicio tardío; los casos en los que se encuentra un IP alterado tienen 6 veces más riesgo de cesárea de emergencia comparado con aquellos con IP normal (38). La sensibilidad de la medición de este vaso es de $7,7 \%$ con especificidad de $90 \%$, valor predictivo positivo de $87,5 \%$ y valor predictivo negativo de $9,78 \%$ (39).

Relación cerebro-placenta: Esta medida se ha tomado como predictor de estancia en UCI neonatal, bajos puntajes de APGAR, valores de $\mathrm{pH}$ de sangre del cordón, cesáreas por sufrimiento fetal y otras complicaciones, es calculada por una división entre los índices de pulsatilidad de la arteria cerebral media y la arteria umbilical. La relación cerebro/placenta representa la interacción de las alteraciones del flujo sanguíneo cerebral que se manifiestan como un aumento en el flujo diastólico como resultado de la vasodilatación de la arteria cerebral media secundario a la hipoxia y el aumento de la resistencia placentaria que se manifiesta como una 
disminución del flujo sanguíneo en diástole en la arteria umbilical $(38,39)$. Recientemente se ha reconocido esta relación como el indicador más sensible y específico para la detección de resultados adversos perinatales en embarazos de alto riesgo y es mejor predictor que el IP de la arteria cerebral media o el IP de la arteria umbilical cuando se usan por separado $(39,41,42)$.

Cuando se encuentra alteración en la relación cerebro/placenta en fetos a término, hay un aumento significativo en el porcentaje de estado fetal no satisfactorio durante el trabajo de parto $(79,1 \%$ vs $10,7 \%$ $\mathrm{p}<0,001)(43,44)$, requiriendo cesáreas de emergencia, adicionalmente estos fetos tienen un menor $\mathrm{pH}$ en sangre de cordón (7.17 vs 7.25 p<0,001), mayores admisiones a UCI neonatal $(11,25 \%$ vs $5,6 \%$ $\mathrm{p}<0,03)(43,44)$. La sensibilidad de esta medida para la detección de resultados adversos es de 68,8\%, especificidad de $100 \%$, valor predictivo positivo de $100 \%$ y valor predictivo negativo de $26,3 \%$ (39).

Ductus venoso: La evaluación del ductus venoso refleja el estado fisiológico del ventrículo derecho e incluye dos picos de onda: el primero de ellos refleja la sístole ventricular, el segundo pico refleja el llenado pasivo en la diástole ventricular $(34,41)$. La onda a corresponde a la contracción auricular en la diástole (Figura 4) y ante el compromiso hemodinámico del feto, esta onda presenta cambios como flujo ausente o reverso $(45,46)$. Estos cambios se encuentran relacionados con el aumento de la poscarga del ventrículo derecho y falla cardiaca derecha ocasionada por hipoxia del miocardio, y se ha documentado en fetos con RCIU asociándose a tasas de mortalidad aumentadas $(45,46)$. Se ha demostrado que los flujos en el ductus venoso se vuelven anormales solo en estados avanzados de compromiso fetal (38). Flujos ausentes o reversos en la onda a se asocian con mortalidad perinatal independientemente de la edad gestacional al momento del parto con un riesgo del $40-100 \%$ en RCIU temprano; en el $50 \%$ de los casos estos cambios preceden la pérdida de variabilidad a corto plazo en el registro cardiotocográfico y en el $90 \%$ de los casos es anormal 48 a 72 horas antes que el perfil biofísico $(38,47)$.

Istmo aórtico: Este vaso refleja el balance entre la impedancia del cerebro y el sistema vascular. El aumento en la resistencia placentaria puede llevar a la disminución del flujo diastólico, y en casos más severos flujo reverso en diástole siendo un signo de deterioro avanzado $(38,48)$. Es por esto que la monitorización del patrón de flujo a este nivel puede jugar un papel importante en la prevención de secuelas neurológicas posnatales ocasionadas por la lesión hipóxica, incluso, puede brindar datos de forma más temprana que algunos marcadores agudos como el ductus venoso (cuando hay alteraciones a este nivel, ya hay acidemia y necrosis miocárdica) (49-52).

Evaluación de la función cardiaca fetal: La
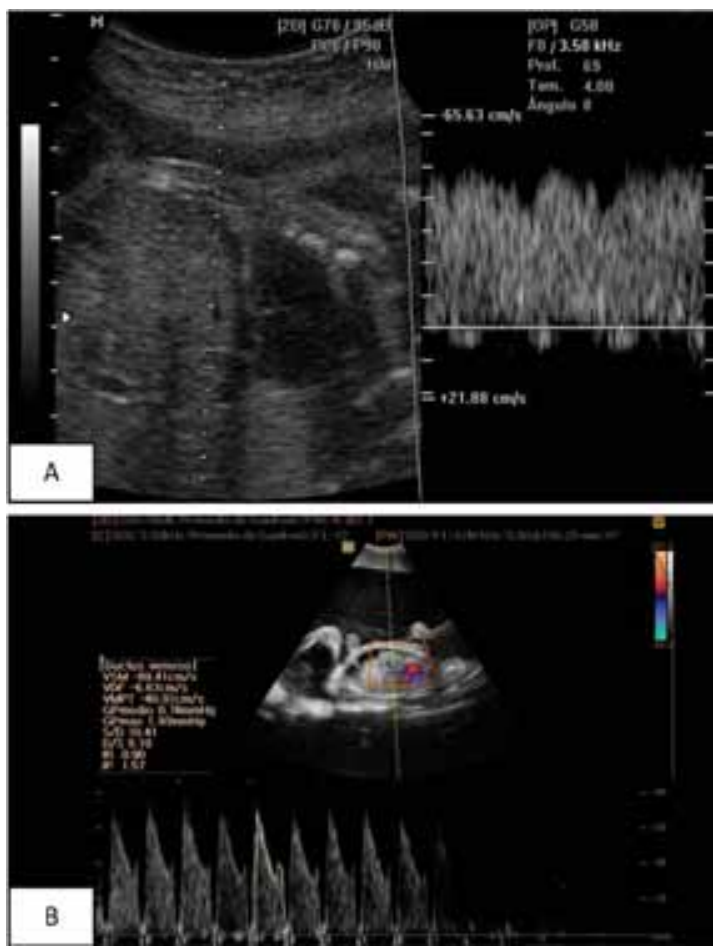

Figura 4. Doppler del ductus venoso. A: Patrón de flujo normal en el ductus venoso en el que se observan los picos de sístole ventricular, diástole precoz y la onda a que refleja la contracción auricular. B: Patrón de flujo anormal en el ductus venoso con flujo reverso de la onda a.

evaluación de la función cardiaca fetal provee información importante del estado hemodinámico. La evaluación de los tractos de salida permite obtener información que permite el cálculo del gasto cardiaco. En fetos normales, el ventrículo derecho contribuye con aproximadamente el $60 \%$ del gasto cardiaco, pero en fetos con RCIU, se invierte esta proporción, siendo mayor el aporte del ventrículo izquierdo ya que se debe aumentar el flujo sanguíneo a la parte superior del cuerpo y cerebro $(47,48)$.

\section{MANEJO}

Se pueden encontrar tres escenarios en estos fetos: A. Con menos de 26 semanas de gestación con una mortalidad mayor del $90 \%$. B. Entre 26 y 28 semanas con tasas de mortalidad entre $30-40 \%$. C. Mayor de 28 semanas, que en estos casos la mortalidad disminuye a menos del 10\% $(51,53)$. La clasificación del Doppler en estadíos de acuerdo a la historia natural de los cambios en la circulación fetal ayuda al seguimiento y a la toma de decisiones (Tabla I) (Figura 5). 
Tabla I

ESTADÍOS DE LA EVALUACIÓN DOPPLER EN FETOS CON RCIU

\begin{tabular}{|c|c|}
\hline Clasificación & Características \\
\hline Doppler tipo I & $\begin{array}{l}\text { PFE }<\text { P } 3 \\
\text { - Relación Cerebro/Placenta }<\text { P5 } \\
\text { - IP arteria umbilical > P } 95 \\
\text { - IP de ACM < P5 } \\
\text { - IP de arterias Uterinas > P } 95\end{array}$ \\
\hline Doppler Tipo II & $\begin{array}{l}\text { PFE }<\text { P } 10 \text { con alguno de los siguientes: } \\
\text { - Flujo diastólico ausente en arteria umbilical (>50\% de ciclos en asa libre en ambas } \\
\text { arterias, en dos ocasiones }>12 \text { horas) } \\
\text { - Flujo diastólico reverso en el istmo aórtico }\end{array}$ \\
\hline Doppler Tipo III & $\begin{array}{l}\text { PFE }<\text { P } 10 \text { con alguno de los siguientes: } \\
\text { - Flujo diastólico reverso en la arteria umbilical (>50\% de ciclos en asa libre en ambas } \\
\text { arterias, en dos ocasiones }>12 \text { horas) } \\
\text { - IP del ductus venoso > P } 95 \text {. }\end{array}$ \\
\hline Doppler Tipo IV & $\begin{array}{l}\text { PFE }<\text { P } 10 \text { con alguno de los siguientes: } \\
\text { - Registro cardiotocográfico patológico (variabilidad }<5 \text { latidos/minuto y/o patrón de } \\
\text { desaceleraciones) } \\
\text { - Flujo diastólico reverso en el ductus venoso }\end{array}$ \\
\hline
\end{tabular}

PFE: Peso fetal estimado. P: Percentil. IP: Índice de pulsatilidad. ACM: Arteria cerebral media.

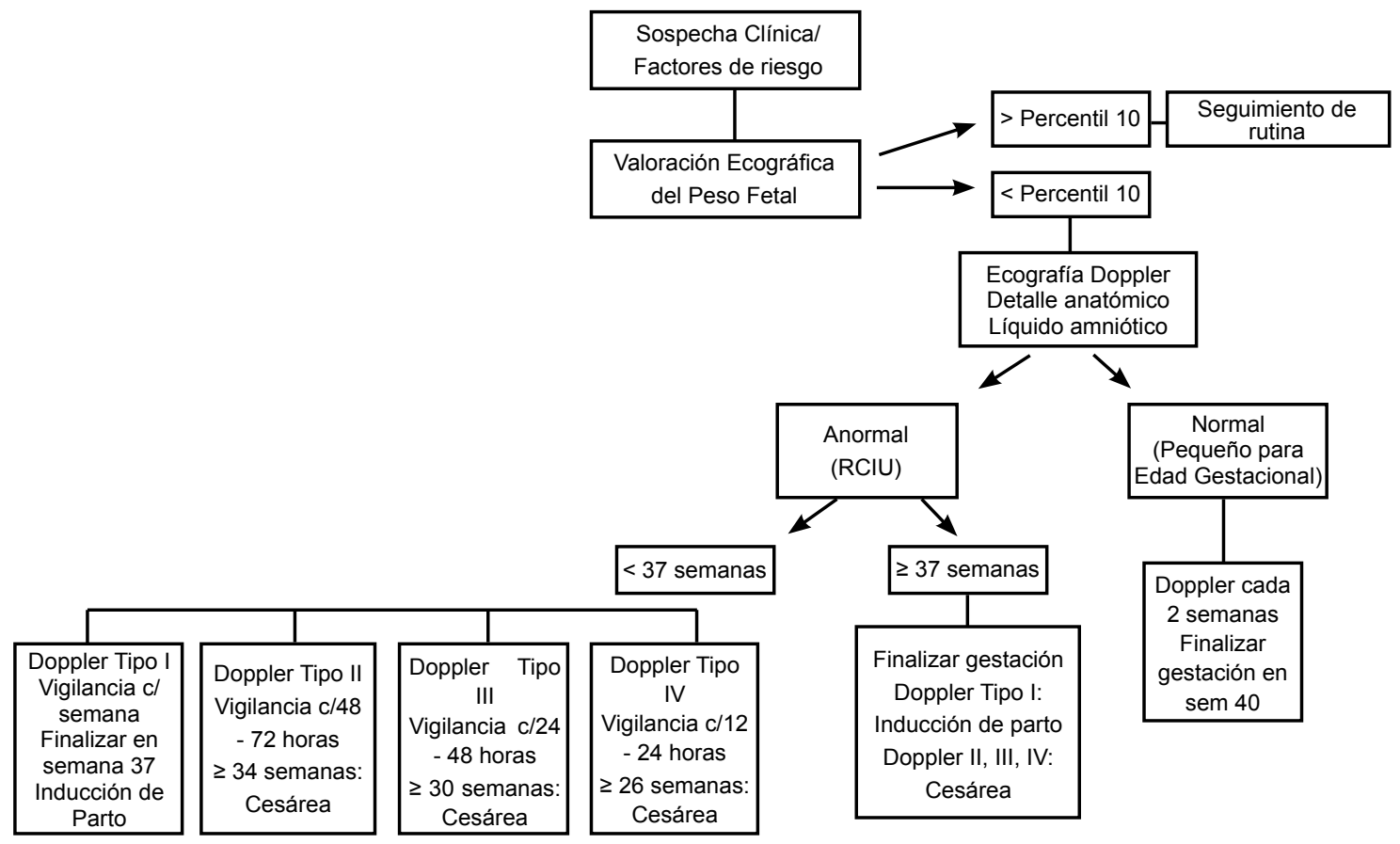

Figura 5. Algoritmo de manejo de fetos con RCIU. 
En la RCIU de aparición tardía la principal característica es la no alteración del flujo en la arteria umbilical, en estos casos la fisiopatología es diferente ya que el porcentaje de lesión placentaria es menor, la hipoxia es mínima, no hay adaptación cardiovascular sistémica y la tolerancia a la hipoxia es menor por lo que la fase de descompensación se da con cambios mínimos (54). Estos fetos tienen peores resultados perinatales por lo tanto deben diagnosticarse y tratarse a tiempo (54). Los principales marcadores que indican pobre pronóstico perinatal son alteraciones en el Doppler de arterias uterinas, relación cerebro/placenta y percentil de crecimiento $<3(38,55-57)$.

El seguimiento debe incluir la arteria cerebral media y arteria umbilical con el fin de construir la relación cerebro/placenta. Cuando hay signos de insuficiencia placentaria (relación cerebro placenta alterada o Doppler de arterias uterinas patológico) el control es cada semana. Ante la evidencia de vasodilatación cerebral se hace el control 2 veces por semana y además se debe añadir un marcador agudo (ductus venoso, registro cardiotocográfico) (38).

En estos fetos es razonable la finalización de la gestación entre 37 y 38 semanas y no se contraindica el parto vaginal $(38,55)$. Aproximadamente en el $20 \%$ hay alteración de la relación cerebro/ placenta (percentil <5), en otro $20 \%$ se puede encontrar alteración de la arteria cerebral media (percentil $<5$ ) y en $20 \%$ se pueden encontrar signos de insuficiencia placentaria que se manifiestan en la cara materna (Doppler de arteria umbilical percentil $>95$ ), cada uno de estos signos se encuentra asociado a pobres resultados perinatales $(49,59)$.

La evaluación en los RCIU tempranos se puede realizar con pruebas que marcan alteraciones crónicas como son el Doppler de arterias uterinas, de arteria umbilical y cerebral media, que se alteran de forma progresiva $(38,58)$.

El control de los fetos que presentan signos de insuficiencia placentaria, debe ser realizado semanalmente, evaluándose arteria umbilical y cerebral media como marcadores crónicos (38).

Si se encuentran signos de insuficiencia placentaria severa o signos de hipoxia (vasodilatación cerebral) el control debe realizarse 2 veces por semana, evaluando marcadores agudos (ductus venoso, registro cardiotocográfico computarizado) además de función cardiaca $(38,59,60,61)$.

Ante signos de acidosis como patrón de flujo reverso en arteria umbilical, alteración en los flujos venosos o alteraciones en el registro cardiotocográfico, el control debe ser más frecuente, realizándose cada 12-24 horas e incluyendo por lo menos dos pruebas agudas $(38,60,61)$.

En cuanto al momento del parto en fetos con restricción del crecimiento precoz, la decisión se debe tomar con base a los diferentes escenarios clínicos: A. Cuando hay insuficiencia placentaria severa (flujo diastólico ausente de arteria umbilical o flujo reverso en el istmo aórtico): finalizar la gestación en semana 34, la vía del parto en estos casos es la cesárea electiva, estos fetos tienen reserva placentaria limitada y no toleran el estrés que produce el parto, lo que resulta en mayores tasas de cesáreas de urgencias $(38,59,61)$. B. En los casos en lo que se encuentren signos de acidosis (flujo diastólico reverso en la arteria umbilical, IP del ductus venoso percentil>95) el riesgo de muerte a las 30 semanas es mayor que los riesgos que trae consigo la prematuridad, por lo tanto estas gestaciones se terminan en esta semana (38). C. En los fetos en los que se encuentra alteración en los flujos venosos antes de las 30 semanas o con alta sospecha de acidosis fetal (flujo reverso en ductus venoso o alteraciones en registro cardiotocográfi$\mathrm{co})$, se recomienda finalizar gestación en semana $28(38,59,61)$. D. Por debajo de las 28 semanas, la tasa de supervivencia es muy baja (menos del $50 \%$ ), por lo tanto, la decisión de finalizar la gestación se justifica ante la presencia de flujo reverso en ductus venoso o de un patrón de desaceleración en el monitoreo. La vía del parto en estos casos es la cesárea ya que estos fetos toleran mal el parto vaginal (38).

\section{CONCLUSIONES}

La RCIU es una patología de diagnóstico frecuente y que representa grandes tasas de morbimortalidad perinatal y secuelas a corto y largo plazo; por lo tanto es importante la realización de un diagnóstico adecuado y a tiempo, además de un seguimiento estricto con el fin de prevenir complicaciones. La ecografía Doppler es una herramienta de vital importancia utilizada en el diagnóstico y seguimiento de los fetos con RCIU, identificándose cada vez más marcadores tempranos que brindan información pronóstica que debe ser utilizada para disminuir los resultados adversos a corto y largo plazo, siendo la relación cerebro/placenta el marcador que actualmente mejor se correlaciona con este tipo de resultados. La propuesta actual de realizar un protocolo de seguimiento y manejo basados en la clasificación del RCIU por estadios brinda facilidad en cuanto a la toma de decisiones, principalmente a la hora de finalizar la gestación y disminuye a la vez la variabilidad del manejo que se observa en la práctica clínica.

\section{REFERENCIAS}

1. Sanín-B. JE, Gómez Díaz J, Ramirez J, Mejía C, Medina O, Vélez J. Consenso nacional de expertos. Diagnóstico y seguimiento del feto con Restricción del crecimiento intrauterino (RCIU) y del feto pequeño para la edad gestacional (PEG). Consenso colombiano. Rev Colomb Obstet Ginecol 2009;60(3):247-61. 
2. Palloto E, Kilbride H. Perinatal outcome and later implications of intrauterine growth restriction. Clin Obstet Gynecol 2006;49(2):257-69.

3. Olusanya B. Intrauterine growth restriction in a lowincome country: Risk factors, adverse perinatal outcomes and correlation with current WHO Multicenter Growth Reference. Early Hum Dev 2010;86:439-44.

4. Garite TJ, Reese C, Thorp J. Intrauterine growth restriction increases morbidity and mortality among premature neonates. Am J Obstet Gynecol 2004;191:481-7.

5. Egaña-Ugrinovic G, Sanz-Cortes M, Figueras F, Bargalló N, Gratacós E. Differences in cortical development assessed by fetal $\mathrm{MRI}$ in late-onset intrauterine growth restriction. Am J Obstet Gynecol 2013;209:126.e1-8.

6. Padilla N, Falcón C, Sanz-Cortés M, Figueras F, Bargallo N, Crispi F, Eixarch E, Arranz A, Botet F, Gratacós $\mathrm{E}$. Differential effects of intrauterine growth restriction on brain structure and development in preterm infants: A magnetic resonance imagings tudy. Brain Res 2011;1382:98-108.

7. Maulik D. Fetal growth compromise: definitions, standards, and classification. Clin Obstet Gynecol 2006;49(2):214-8.

8. Gardosi J. Intrauterine growth restriction: new standards for assessing adverse outcome. Best Pract Res CI OB 2009;23:741-9.

9. Baschat A. Fetal growth disorders. En: James DK, Steer PJ, Weiner CP, Gonik B. High risk obstetrics: management options. 3rd ed. Elsevier editorial; 2005. pp. 240-72.

10. Haram K, Softeland E, Bukowski R. Intrauterine growth restriction. Int J Gynecol Obstet 2006;935-12.

11. Royal College of Obstetricians and Gynaecologists (RCOG). The investigation and management of the small for gestational age fetus. RCOG. Green Top guideline № 31 London. UK.

12. Maulik D. Fetal Growth Restriction: The Etiology. Clin Obstet Gynecol2006;49(2):228-35.

13. Salafia C, Charles A, Maas E. Placenta and fetal growth restriction. Clin Obstet Gynecol 2006;49(2):236-56.

14. Hendrix N, Berghella V. Non-placental causes of intrauterine growth restriction. Semin Perinatol 2008;32:161-5.

15. Chauhan S, Magann E. Screening for fetal growth restriction. Clin Obstet Gynecol 2006;49(2):284-94.

16. Odegard RA, Vatten LJ, Nilsen ST, Salvesen KA, Austgulen R. Preeclampsia and fetal growth. Obstet Gynecol 2000;96:950-5.

17. Sibai BM, Spinnato JA, Watson DL, Hill GA, Anderson GD. Pregnancy outcome in 303 cases with severe preeclampsia. Obstet Gynecol 1984;64:319-25.

18. Sibai BM. Chronic hypertension in pregnancy. Obstet Gynecol 2002;100:369-77.

19. Howley HE, Walker M, Rodger MA. A systematic review of the association between factor $\mathrm{V}$ Leiden or prothrombin gene variant and intrauterine growth restriction. Am J Obstet Gynecol 2005;192:694-708.

20. Cliver SP, Goldenberg RL, Cutter GR, Hoffman HJ, Davis RO, Nelson KG. The effect of cigarette smoking on neonatal anthropometric measurements. Obstet Gynecol 1995;85:625-30.

21. Cnattingius S, Mills JL, Yuen J, Eriksson O, Salonen $H$. The paradoxical effect of smoking in preeclamptic pregnancies: smoking reduces the incidence but increases the rates of perinatal mortality, abruption placentae, and intrauterine growth restriction. Am J Obstet Gynecol 1997;177:156-61.

22. Mook-Kanamori DO, Steegers EA, Eilers PH, Raat $H$, Hofman A, Jaddoe, V. Risk factors and outcomes associated with first trimester fetal growth restriction. JAMA 2010;303(6):527-34.

23. Bada HS, Das A, Bauer CR, Shankarah S, Lester B, Wright LL, et al. Gestational cocaine exposure and intrauterine growth: maternal lifestyle study. Obstet Gynecol 2002;100:916-24.

24. Grote N, Bridge J, Gavin A, Melville J, Lyengar S, Katon W. A metaanalysis of depression during pregnancy and the risk of preterm birth, low birth weight and intrauterine growth restriction. Arch Gen Psychiatry 2010;67(10):1012-24.

25. Monk D, Moore G. Intrauterine growth restriction genetic causes and consequences. Semin Fetal Neonatal Med 2004;9:371-8.

26. Ville Y, Nyberg DA. Growth, Doppler and fetal assessment. En: Nyberg DA, McGahan JP, Pretorius $\mathrm{DH}$, Pilu G, editors. Diagnostic imaging of fetal anomalies. Philadelphia: Lippincott Williams \& Wilkins; 2003. pp 31-58.

27. Offenbacher S, Lieff S, Boggess KA, Murtha AP, Madianos PN, Champagne CM, et al. Maternal periodontitis and prematurity. Part I: Obstetric outcome of prematurity and growth restriction. Ann Periodontol 2001;6:164-74.

28. Kumar A, Basra M, Begum N, Rani V, Prasad S, Lamba AK, et al. Association of maternal periodontal health with adverse pregnancy outcome. J Obstet Gynaecol Res 2013;39(1):40-5.

29. Abati S, Villa A, Cetil I, Dessole S, Lugliè PF, Strohmenger $\mathrm{L}$, et al. Lack of association between maternal periodontal status and adverse pregnancy outcomes: a multicentric epidemiologic study. J Matern Fetal Neonatal Med 2013;26(4):369-72.

30. Gardosi JO. Prematurity and fetal growth restriction. Early Hum Dev 2005;81:43-9.

31. Bukowski R, Gahn D, Denning J, Saade G. Impairment of growth in fetuses destined to deliver preterm. Am J Obstet Gynecol 2001;185:463-7.

32. Kovo M, Schriber L, Ben-Haroush A, Cohen G, Weiner E, Golan A, Bar J. The placental factor in earlyand late-onset normotensive fetal growth restriction. Placenta 2013;34: 320-4.

33. Harman C. Baschat A. Arterial and Venous Dopplers in IUGR. Clin Obstet Gynecol 2003;46(4):931-46.

34. Baschat $A$. The fetal circulation and essential organs - a new twist to and old tale. Ultrasound Gynecol 2006;27:349-54.

35. Marsal K. Obstetric management of intrauterine growth restriction. Best Prac Res CI OB 2009;23:857-70.

36. Cruz-Martinez R, Savchev S, Cruz-Lemini M, Mendez $A$, Gratacos E, Figueras F. Clinical utility of third-trimester uterine artery Doppler in the prediction of brain hemodynamic deterioration and adverse perinatal outcome in small for-gestational-age fetuses. Ultrasound Obstet Gynecol 2015;45(3):237-8.

37. Contro E, Cha DH, De Maggio I, Ismail SY, Falcone V, Gabrielli S, Farina A. Uterine artery Doppler longitudinal changes in pregnancies complicates with intrauterine growth restriction without preeclampsia. Prenat Diagn 2014;34(13):1332-6. 
38. Figueras F, Gratacós E. Update on the diagnosis and classification of fetal growth restriction and proposal of a stage-based management protocol. Fetal Diagn Ther 2014;36(2):86-9.

39. Malik R, Saxena A. Role of color Doppler indices in the diagnosis of intrauterine growth retardation in high-risk pregnancies. J Obstet Gynaecol India 2013;63(1):37-44.

40. Berkley E, Chauhan S, Abuhamad A. Doppler assessment of the fetus with intrauterine growth restriction. Am J Obstet Gynecol 2012;206(4):300-8.

41. Figueroa-Diesel H, Hernandez-Andrade E, AcostaRojas R, Cabero L, Gratacos E. Doppler changes in the main fetal brain arteries at different stages of hemodynamic adaptation in severe intrauterine growth restriction. Ultrasound Obstet Gynecol 2007;30(3):297302.

42. Cruz - Martinez, R. Figueras F. The role of Doppler and placental screening. Best Prac Res Cl Ob 2009; 23:845-55.

43. DeVore G. The importance of the cerebroplacental ratio in the evaluation of fetal wellbeing in SGA and AGA fetuses.Am J Obstet Gynecol 2015;213(1):5-15.

44. Figueras F, Savchev S, Triunfo S, Crovetto F, Gratacós $E$. An integrated model with classification criteria to predict small-for-gestational-age fetuses at risk of adverse perinatal outcome. Ultrasound Obstet Gynecol 2015; 45(3):279-85.

45. Odibo AO, Riddick C, Pare E, Stamilio DM, Macones GA. Cerebroplacental Doppler ratio and adverse perinatal outcomes in intrauterine growth restriction: evaluating the impact of using gestational age-specific reference values. J Ultrasound Med 2005;24(9):1223-8.

46. Hernandez-Andrade E, Crispi F, Benavides-Serralde A, Plasencia W, Diesel HF, Eixarch E, et al. Contribution of the myocardial performance index and aortic isthmus blood flow index to predicting mortality in preterm growth-restricted fetuses. Ultrasound Obstet Gynecol 2009;34:430-6.

47. Turan O, Turan S, Berg C, Gembruch U, Nicolaides $\mathrm{KH}$, Harman CR, Baschat AA. Duration of persistent abnormal ductus venous flow and its impact on perinatal outcome in fetal growth restriction. Ultrasound Obstet Gynecol 2011;38:295- 302.

48. Acharya G, Tronnes A, Rasanen J. Aortic isthmus and cardiac monitoring of the growth-restricted fetus. Clin Perinatol 2011;38:113-25.

49. Hernandes-Andrade E, Benavides-Serralde J, CrozMartinez R, Welsh A, Mancilla-Ramirez J. Evaluation of conventional doppler fetal cardiac function parameters: E/A ratios, outflow tracts, and myocardial performance index. Fetal Diagn Ther 2012;32:22-9.

50. Fouron JC, Gosselin J, Raboisson MJ, Lamoureux J, Tison CA, Fouron C, Hudon L. The relationship between an aortic isthmus blood flow velocity index and the postnatal neurodevelopmental status of fetuses with placental circulatory insufficiency. Am J Obstet Gynecol 2005;192:497-503.

51. Cruz-Martinez R, Figueras F, Benavides-Serralde A, Crispi F, Hernandez-andrade E, Gratacos E. Sequence of changes in myocardial performance index in relation to aortic isthmus and ductus venosus Doppler in fetuses with early-onset intrauterine growth restriction. Ultrasound Obstet Gynecol 2011;38:179-84.

52. Cruz-Lemini M, Crispi Fátima, Van Mieghem T, Pedraza D, Cruz-Martinez R, et al. Risk of perinatal death in early-onset intrauterine growth restriction according to gestational age and cardiovascular doppler indices: a multicenter study. Fetal DiagnTher 2012;32:116-22.

53. Oros D, Figueras F, Cruz-Martinez R, Meler E, Munmany $\mathrm{M}$, Gratacos $\mathrm{E}$. Longitudinal changes in uterine, umbilical and fetal cerebral Doppler indices in lateonset small-for-gestational age fetuses. Ultrasound Obstet Gynecol 2011;37:191-5.

54. Savchev S, Flgueras F, Cruz.Martinez R, Illa M, Botet $F$, Gratacos E. Estimated weight centile as a predictor of perinatal outcome in small-for gestational-age pregnancies with normal fetal and maternal Doppler indices. Ultrasound Obstet Gynecol 2012;39(3):299303.

55. Boers KE, Vijen SM, Bijenga D, Van der Post JA, Bedekam DJ, Kwee A. Induction versus expectant monitoring for intrauterine growth restriction at term: randomized equivalence trial (DIGITAT). BMJ 2010;341:c7087.

56. Galan H. Timing delivery of the growth-restricted fetus. SeminPerinatol 2011;35(5):262-9.

57. Figueras F, Gardosi J. Intrauterine growth restriction: new concepts in antenatal surveillance, diagnosis, and management. Am J Obstet Gynecol 2011;204:288300.

58. Baschat A, Cosmi E, Bilardo CM, Wolf $\mathrm{H}$, Berg C, Rigano $\mathrm{S}$, et al. Predictors of neonatal outcome in early- onset placental dysfunction. Obstet Gynecol 2007;109(2 Pt 1):253-61.

59. The GRIT Study Group. When do obstetricians recommend delivery for a high-risk preterm growthretarded fetus? Eur J Obstet Gynecol Reprod Biol 1996;67:121-6.

60. Sterne G, Shields LE, Dubinsky TJ. Abnormal fetal cerebral and umbilical Doppler measurements in fetuses with intrauterine growth restriction predict the severity of perinatal morbidity. J Clin Ultrasound 2001;29:14651.

61. Grivell RM, Alfirevic Z, Gyte GM, Devane D. Antenatal cardiotocography for fetal assessment. Cochrane Database Syst Rev 2012;12:CD007863. 